expressed ISG gene set in IGHM+, IGHD+, and IGHG + B cells in AA patients with autoantibodies and renal disease. Further, ISG highly expressing SLE B cells exhibited unique heavy- and light-chain repertoires including expression of the autoreactive IGHV4-34 gene, targeted with the 9 G4 anti-idiotype antibody that recognizes DNA- and RBP-autoreactive B cells.

Conclusions (i) B cells are an important source of type I IFNs in modulating TLR and BCR responses in SLE; (ii) there are well-orchestrated distinct programs in type I IFN expression and response genes in subsets of B cells, (iii) distinct pathways of autoreactive B cell survival and activation are effected by combined signaling through BCR, TLR, and IFNAR with resultant distinct BCR heavy- and light-chain repertoire.

Funding Source(s): R01-AI-071110, R01 AI134023, LRA Distinguished Innovator Award and Novel Research Award, VA Merit Review Award I01B × 004049, Immunology T32 Training Grant 2T32AI007051-39, the LFA Finzi Summer Fellowship.

\section{THE ENVIRONMENT AND LUPUS: IMPACT OF INFLAMMATION AND DIET ON T CELL EPIGENETICS}

Bruce C Richardson. University of Michigan

\subsection{6/lupus-2019-Ism.193}

Background Lupus flares when genetically predisposed people encounter environmental agents that trigger the flares such as UV light and infections which cause oxidative stress, but the mechanisms by which environmental agents induce flares are unclear. Our group has shown that lupus-inducing drugs such as procainamide and hydralazine inhibit DNA methylation in dividing CD4 + T cells, converting normal antigen specific $\mathrm{T}$ cells into autoreactive, cytotoxic pro-inflammatory cells that are sufficient to cause lupus in mice, and that similar epigenetically altered $\mathrm{T}$ cells are found in patients with active lupus. The mechanism(s) by which environmental agents alter the $\mathrm{T}$ cell epigenome to create the pathogenic cells was unclear. The enzyme DNA methyltransferase 1 (Dnmt1) is upregulated as $\mathrm{T}$ cells enter mitosis by signals transmitted through the ERK pathway, then binds the replication fork where it copies methylation patterns from the parent strand to the daughter strand by transferring the methyl group from S-adenosylmethionine (SAM) to $\mathrm{dC}$ bases in the daughter strand. This suggests that environmental agents which inhibit Dnmt1 upregulation or decrease SAM levels may inhibit $\mathrm{T}$ cell DNA methylation to trigger lupus flares.

Methods CD4 + T cells from lupus patients and controls were stimulated with PHA then cultured in custom media with normal or low transmethylation micronutrient levels. Oxidative stress was induced by treating the normal CD4 $+\mathrm{T}$ cells with peroxynitrite (ONOO-) prior to culture or injection into SJL mice. Methylation sensitive gene expression (CD70, KIR, and perforin) was measured by RT-PCR and flow cytometry

Results PHA stimulated CD4 + T cells from healthy controls expressed higher levels of CD70, KIR, and perforin mRNA and protein when cultured in media with low transmethylation micronutrient levels relative to cells cultured in complete media. Similar increases were seen in cells cultured in media with low or normal media methionine levels. PHA stimulated CD4 + T cells from lupus patients also overexpressed KIR, CD70 and perforin relative to PHA stimulated T cells from controls when similarly cultured. Treating PHA stimulated normal CD4 + T cells with ONOO- also increased methylation sensitive gene expression in normal CD4 $+\mathrm{T}$ cells, and low methionine or folate levels further increased gene expression relative to untreated $\mathrm{T}$ cells and $\mathrm{T}$ cells cultured in complete tissue culture media.

Conclusions Inflammation and transmethylation micronutrient deficiencies synergize to inhibit T cell DNA methylation, contributing to the onset of lupus flares.:

Funding Source(s): Lupus Insight Prize from the Alliance for Lupus Research, the Lupus Research Institute and the Lupus Foundation of America

\section{TRANSCRIPTOME ANALYSIS OF SKIN FIBROBLASTS DERIVED FROM LUPUS NEPHRITIS PATIENTS DEMONSTRATES FIBROTIC AND INTERFERON-RELATED CELLULAR BIOMARKERS}

${ }^{1}$ Robert Clancy*, ${ }^{2}$ Hemant Suryawanshi, ${ }^{1}$ Michael Belmont, ${ }^{1}$ Peter Izmirly, ${ }^{2}$ Thomas Tuschl, ${ }^{1}$ Jill Buyon. ${ }^{1}$ NYU School of Medicine; ${ }^{2}$ The Rockefeller University

10.1136/lupus-2019-Ism.194

Background The impact of renal injury in lupus nephritis is widespread with consequences to resident cells in other tissue beds, even non-lesional non-sun exposed skin. Faithful reflection of a relevant renal tissue pathway in a more readily accessible compartment would allow for less invasive diagnostic alternatives. Single-cell transcriptional states as performed in this study may provide a framework for understanding how in vivo biological function emerges from complex cell ensembles, thus allowing for a clearer understanding of potential mutual pathways.

Methods Patients with proteinuria and known ISN/RPS Class and controls were recruited to discovery 1 and 2 cohorts. Single cell RNAseq was performed on cell suspensions prepared from $\sim 2 \mathrm{~mm}$ punch biopsies of non-lesional non sun-exposed skin from the buttocks. The libraries were prepared on the Fluidigm C1 platform (discovery 1) and 10X Genomics platform (discovery 2) along with Illumina HiSeq 2500 sequencing.

Results Sorting based on COL1A1, COL1A2, COL3A1, MFAP5 and MFAP4 expression yielded 12 fibroblasts from 3 patients. The 1 Class II subject yielded 5 single-cell transcriptomes. The other 2 subjects (1 Class IV,V; 1 Class III,V) yielded 7 single-cell transcriptomes. 22 transcriptomes were derived from 3 controls. The aggregate data were used to determine the top upregulated genes in cases versus controls, most of which belonged to the interferon-stimulated gene category and the extracellular matrix category (DAVID databases). Fewer cells were obtained using Fluidigm C1 (36 single-cell) than 10X Genomics (7280 single-cell). For the latter, the major biopsy classes were represented (Class III, III/IV, III/V, V and no LN). We applied graph-based clustering and identified 12 major clusters of cells from the patient skin as visualized by $\mathrm{t}$-distributed stochastic neighbor embedding ( $\mathrm{t}$-SNE; figure 1). Differential gene expression analysis guided by established lineage markers revealed three keratinocyte clusters (KC1KC3), two fibroblast clusters (FB1, FB2), smooth muscle cells (SMC), two endothelial cell clusters (VEC, LEC), melanocytes (MEL), sweat gland cells (SG), macrophages/dendritic cells (MAC-DC) and $\mathrm{T}$ cells (TC). Ranked by abundance, patient skin exhibited $\mathrm{KC}>\mathrm{FB}>\mathrm{EC}>\mathrm{MAC}-\mathrm{DC}>\mathrm{SMC}>\mathrm{TC}>\mathrm{SG}>\mathrm{MEL}$. 


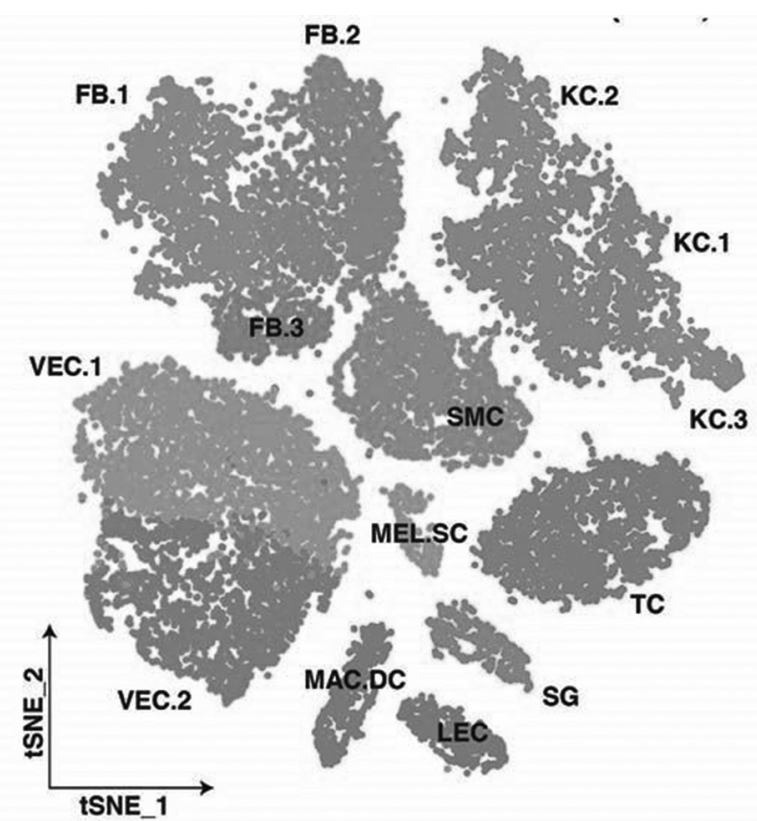

Abstract 194 Figure 1 Major clusters of cells derived from 8 patient skin punch biopsies visualized by tSNE $(n=7280)$. Differential gene expression analysis guided by established lineage markers revealed three keratinocyte clusters (KC1-KC3), two fibroblast clusters (FB1, FB2), smooth muscle cells (SMC), two endothelial cell clusters (VEC, LEC), melanocytes (MEL), sweat gland cells (SG), macrophages/dendritic cells (MAC-DC) and T cells (TC). Ranked by abundance, patient skin exhibited $\mathrm{KC}>\mathrm{FB}>\mathrm{EC}>\mathrm{MAC}-\mathrm{DC}>\mathrm{SMC}>\mathrm{TC}>\mathrm{SG}>\mathrm{MEL}$

Conclusions Single-cell RNAseq is both feasible and informative in cell-specific transcriptome analysis of fresh non-lesional non-sun exposed LN skin biopsies. 10X genomics significantly increases cell numbers and facilitates identification of major cell clusters compared to Fluidigm C1. The expression of fibroblasts and genes reflective of fibrotic and interferonrelated pathways support the application of this approach to study readily accessible tissue for biomarkers related to disease progression.

Funding Source(s): Judith and Stewart Colton Center for Autoimmunity at NYU Langone Health

Differential gene expression analysis guided by established lineage markers revealed three keratinocyte clusters (KC1KC3), two fibroblast clusters (FB1, FB2), smooth muscle cells (SMC), two endothelial cell clusters (VEC, LEC), melanocytes (MEL), sweat gland cells (SG), macrophages/dendritic cells (MAC-DC) and T cells (TC). Ranked by abundance, patient skin exhibited $\mathrm{KC}>\mathrm{FB}>\mathrm{EC}>\mathrm{MAC}-\mathrm{DC}>\mathrm{SMC}>\mathrm{TC}>\mathrm{SG}>\mathrm{MEL}$.

\section{SEQUENTIAL BLOCKADE OF OX40L FOLLOWED BY PD-1 ALTERED THE SUPPRESSIVE FUNCTION AND PROLIFERATION OF CD4+ REGULATORY T CELLS IN LUPUS MICE}

${ }^{1}$ Maida Wong*, ${ }^{2}$ Bevra H Hahn. ' University of California Irvine/VA Healthcare System Long Beach; ${ }^{2}$ University of California, Los Angeles

\subsection{6/lupus-2019-Ism.195}

Background In systemic lupus erythematosus (SLE), dysregulated production of autoantibodies is a consequence of disrupted $\mathrm{T}$ cell homeostasis. We have published that blockade of
Programmed death-1 (PD-1), a negative regulator in $\mathrm{T}$ cells, limits helper $\mathrm{T}$ cell $(\mathrm{Th})$ activation, restores regulatory $\mathrm{T}$ cell (Treg) suppression, and reinstates immune cell function. We have also shown that attenuated PD-1 expression in Treg down-regulates OX40L, which helps restore the suppressive capacity of Treg. However, concurrent administration of different immunotherapies may negate positive outcomes. We hypothesize that the sequence of blocking PD-1 and OX40L influences the induction and sustainability of Treg suppressivity. Methods We treated 8-week-old BWF1 mice with a neutralizing $\mathrm{Ab}$ against $\mathrm{PD}-1$ or $\mathrm{OX} 40 \mathrm{~L}$ intraperitoneally. At age 25 weeks when anti-dsDNA began to rise, mice were treated with either anti-PD1 or anti-OX40L that they had not received at age 8 weeks. OX40L, Foxp3 and PD-1 expression on CD4 +CD25+Treg from spleens, apoptosis of Treg and CD4 +CD25 Th were measured by flow cytometry. Seum production of IFN (Th1), IL4 (Th2), IL17a (Th17) and TGF(Treg), and anti-dsDNA (B cells) were measured by ELISA. The survival of these mice were compared to those treated with anti-PD1 alone at 8 weeks, which we previously demonstrated prolonged survival with delayed onset of proteinuria. Results Anti-OX40L suppressed Th function and proliferation independent of Foxp3 expression in Treg with decreased antidsDNA production. Subsequent blockade of PD-1 in antiOX40L-treated mice generated more PD1loTreg with increased TGF- production; it sustained Treg suppressivity and delayed onset of proteinuria when compared to mice treated with anti-PD1 alone. Conversely, sequential blockade of antiOX40L in anti-PD1-treated mice did not promote Treg survival and their disease inconsistently progressed: these mice had predominantly PD1hi or PD1-Treg, and antagonistic OX40L could not restore their suppressivity.

Conclusions Effective induction of Treg is associated with low expression of PD-1 and OX40L, which permits Treg to survive and perform cell suppressive function. Combination of Abs targeting OX40L and PD-1 can improve Treg function and survival outcomes, but it is determined by the timing and sequence of $\mathrm{Ab}$ administration: blocking OX40L followed by PD-1 has an additive effect which is not observed when the order of Abs given was reversed. OX40L and PD-1 signaling communicate sequentially with Treg to regulate its suppressive capacity and survival to achieve peripheral tolerance in SLE, suggesting that treatment with one immunotherapy could change the biology of T-cell signaling such that another immunotherapy may lose its efficacy or has unexpected negative outcome.

Funding Source(s): None

\section{IMMUNE PATHOLOGY CAUSED BY INTRINSIC LOSS OF CONTROL IN CD4 T CELLS}

Jeroen Roose*. UCSF, Department of Anatomy

10.1136/lupus-2019-Ism.196

Background Naïve T cells constantly see self, need to avoid spontaneous immunity, yet be primed to react to foreign antigens when an infection occurs. Both CD4 +and CD8+priimary $\mathrm{T}$ cells exhibit tonic signaling (Myers et al., 2017b) and continuous interactions of the TCR with self-p/MHC generate tonic signals (Stefanova et al., 2002). T cells lacking the central adapter molecule LAT cause a spontaneous lymphoproliferative $\mathrm{T}$ helper 2 (TH2) cell syndrome in mice. Thus, LAT 